\title{
From the bottom up - measuring bulk mechanical properties via microtechnology
}

\author{
N. Barbosa ${ }^{a}$, S. Kim, and D.T. Read
}

National Institute of Standards and Technology, Materials Reliability Division, Boulder, Colorado, 80305, United States of America

\section{Introduction}

The micromechanics community has developed a large set of tools for measuring the properties of micro- and nano-scaled materials. These new tools have been successfully applied to a range of materials, most often in thin-film form, to determine the critical length scales below which bulk properties no longer describe the behaviour of materials, and to understand the underlying mechanisms behind the divergent behaviour. These tools, essential for the characterization of smallscale materials, have recently been applied in a new strategy to evaluate the mechanical properties of bulk materials through microsized testing in situations where conventional testing is challenging such as in extreme or harsh environments (e.g., temperature extremes, radiation exposure, corrosive chemistries, etc.). In this work, the initial results from a microtechnology-based tensile technique developed for the evaluation of bulk materials properties via microscale test specimens sectioned from bulk materials are described.

\section{Test Methodology}

The new tensile test method combines silicon-based micromachining for grip fabrication; a combination of sectioning, polishing, and micro-scale electrical discharge machining (EDM) procedures for specimen preparation; and the use of a previously developed system for actuation and sensing of load and displacement.

In this work, a tapered grip scheme [1] was incorporated into a silicon support frame originally designed for evaluating the tensile properties of thin films [2] to grip the specimens. Figure 1 shows the device layout. Specimens, which were manually placed into the grips prior to testing, were cut from bulk stainless steel with a high-speed precision saw, then manually polished to a thickness of approximately $100 \mu \mathrm{m}$. Final thinning and sample shaping were performed via an EDM method.

Tests were performed in a system designed for free-standing, thin-film, microtensile tests [3] consisting of a loading hook with inline load sensing. Digital image correlation was used to measure displacement. The setup can impose displacement rates ranging from $0.004 \mu \mathrm{m} / \mathrm{s}$ to $2 \mu \mathrm{m} / \mathrm{s}$ and force capacities in millinewtons, though the setup can be easily altered to measure larger forces if required.

\footnotetext{
a e-mail : barbosa@boulder.nist.gov

Contribution of NIST, an agency of the US government; not subject to copyright in the United States.
}

This is an Open Access article distributed under the terms of the Creative Commons Attribution-Noncommercial License 3.0, which permits unrestricted use, distribution, and reproduction in any noncommercial medium, provided the original work is properly cited. 


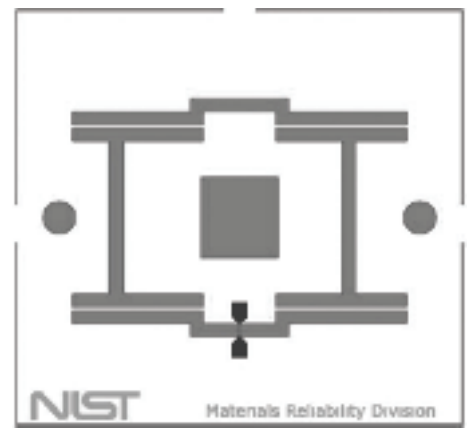

Fig. 1. Schematic showing a single silicon support frame. The medium gray areas show full-thickness wafer etching, and the black areas show partial-thickness etching. The large square hole in the center of the "floating" structure is for external actuation and the round holes are for frame positioning.

Figure 2 shows a silicon support frame with a stainless-steel tensile specimen positioned in the grips. The movement of bottom-right grip provides up to $400 \mu \mathrm{m}$ of displacement to a $400 \mu \mathrm{m}$ gauge section. The grip is part of the "floating" center structure supported by four silicon springs (Fig. 1). The measured stiffness of the springs, $2.1 \pm 0.01 \mathrm{mN} / \mu \mathrm{m}$, agrees with the value $2.7 \mathrm{mN} / \mu \mathrm{m}$ calculated from the as-designed geometry [3]. Error between the values is attributed to differences between the as-designed and as-fabricated geometries. During a tensile test, this stiffness value accounts for the initial loading during the first of two distinct stages seen in the applied force versus displacement curve. As the movable grip "seats" the tensile specimen against the grip faces, the second stage begins and measured applied force now consists of the spring stiffness and the specimen stiffness. This technique has been applied to stainless steel specimens similar to the one shown in Fig. 2. Analysis of the initial stress-strain responses indicate that this technique is a viable option for the testing of bulk materials.

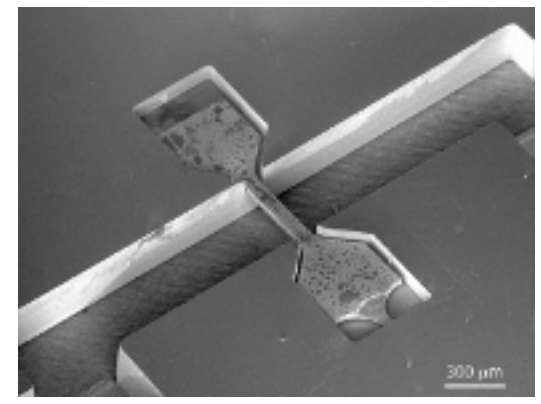

Fig. 2. SEM image of a support frame gripping a stainless steel specimen prepared from bulk sheet.

\section{References}

1. M. Zupan, M.J. Hayden, C.J. Boehlert, K.J. Hemker, Exp. Mech. 21, 242 (2001)

2. J. Gaspar, M.E. Schmidt, J. Held, O. Paul, J. Microelectromech. S. 18, 1062 (2009)

3. Y. Cheng, D.T. Read, J.D. McColskey, J. Wright, Thin Solid Films 484, 426 (2005) 